Pacific Journal of Mathematics

ON THE UNITARY INVARIANCE OF THE NUMERICAL 


\title{
ON THE UNITARY INVARIANCE OF THE NUMERICAL RADIUS
}

\author{
Ivan FilipPenko and Marvin Marcus
}

A characterization is obtained of scalar multiples of unitary matrices in terms of the unitary invariance of a generalized numerical radius. The method of proof involves some rather delicate combinatorial considerations.

1. Introduction. Let $n$ and $m$ be positive integers, $1 \leqq m \leqq$ $n$, and denote by $M_{n, m}(C)\left(M_{n}(C)\right)$ the vector space of all $n$-by- $m$ ( $n$-square) complex matrices. For a matrix $A \in M_{n}(C)$, define the $m$ th decomposable numerical range of $A$ to be the set

$$
W_{m}^{\wedge}(A)=\left\{\operatorname{det}\left(X^{*} A X\right) \mid X \in M_{n, m}(\mathbf{C}), \operatorname{det}\left(X^{*} X\right)=1\right\}
$$

in the complex plane (the reason for this choice of terminology will become apparent in the next section). It is not difficult to verify that $W_{m}^{\wedge}(A)$ is compact, so it makes sense to define the $m$ th decomposable numerical radius of $A$ by

$$
r_{m}^{\hat{m}}(A)=\max _{z \in W_{\hat{m}}(A)}|z|
$$

When $m=1, W_{1}^{\wedge}(A)$ is simply the classical numerical range

$$
W(A)=\left\{(A x, x) \mid x \in \mathbf{C}^{n},\|x\|=1\right\}
$$

(here $(\cdot, \cdot)$ denotes the standard inner product in the space $\mathbf{C}^{n}$ of complex $n$-tuples), and $r_{1}(A)$ is the classical numerical radius

$$
r(A)=\max _{z \in W(A)}|z|
$$

The numerical radius $r(A)$ satisfies the interesting power inequality

$$
r\left(A^{k}\right) \leqq r(A)^{k}, \quad k=1,2,3, \cdots
$$

$[2, \S 176]$. In general, the number $r_{m}^{\wedge}(A)$ is an important function of the matrix $A$. For example, it is a bound for the moduli of all products of $m$ eigenvalues of $A$. This is an immediate consequence of Proposition 1. Another easy consequence (Corollary 2) of Proposition 1 is that if $\boldsymbol{A}$ 
is a scalar multiple of a unitary matrix, then $r_{m}^{\wedge}(A)$ remains invariant under pre- and postmultiplication of $A$ by arbitrary unitary matrices. The purpose of the present paper is to prove that in fact this invariance property characterizes scalar multiples of unitary matrices (Theorem 1).

2. Preliminary notions. The $m$ th Grassmann space over $\mathbf{C}^{n}$, denoted by $\wedge^{m} \mathbf{C}^{n}$, provides an appropriate setting for our investigation of the $m$ th decomposable numerical radius. The standard inner product in $\mathbf{C}^{n}$ induces an inner product in $\wedge^{m} \mathbf{C}^{n}$, given on decomposable symmetrized tensors

$$
x^{\wedge}=x_{1} \wedge \cdots \wedge x_{m}, y^{\wedge}=y_{1} \wedge \cdots \wedge y_{m} \in \wedge^{m} \mathbf{C}^{n}
$$

by

$$
\left(x^{\wedge}, y^{\wedge}\right)=\operatorname{det}\left[\left(x_{i}, y_{\jmath}\right)\right]
$$

The Grassmannian manifold $G_{m}\left(C^{n}\right)$ is the set of all unit length decomposable symmetrized tensors in $\wedge^{m} C^{n}$ :

$$
G_{m}\left(\mathbf{C}^{n}\right)=\left\{x^{\wedge} \in \wedge^{m} \mathbf{C}^{n} \mid\left\|x^{\wedge}\right\|=1\right\}
$$

Let $A \in M_{n}(C)$, and let $C_{m}(A)$ be the $m$ th compound of $A$, so that for $x_{1}, \cdots, x_{m} \in \mathbf{C}^{n}$ we have

$$
C_{m}(A) x_{1} \wedge \cdots \wedge x_{m}=A x_{1} \wedge \cdots \wedge A x_{m} .
$$

If the columns of a matrix $X \in M_{n, m}(\mathrm{C})$ are $x_{1}, \cdots, x_{m}$ in order, then

$$
\operatorname{det}\left(X^{*} A X\right)=\left(C_{m}(A) x_{1} \wedge \cdots \wedge x_{m}, x_{1} \wedge \cdots \wedge x_{m}\right)
$$

Furthermore, $\operatorname{det}\left(X^{*} X\right)=1$ if and only if $x_{1} \wedge \cdots \wedge x_{m} \in G_{m}\left(C^{n}\right)$. Thus from (1),

$$
W_{m}^{\wedge}(A)=\left\{\left(C_{m}(A) x^{\wedge}, x^{\wedge}\right) \mid x^{\wedge} \in G_{m}\left(\mathbf{C}^{n}\right)\right\}
$$

Given $x^{\wedge}=x_{1} \wedge \cdots \wedge x_{m} \in G_{m}\left(C^{n}\right)$, it may in fact be assumed that the vectors $x_{1}, \cdots, x_{m} \in \mathbf{C}^{n}$ are orthonormal [4, p. 1]. Choose, then, a unitary matrix $U \in M_{n}(\mathrm{C})$ such that

$$
U e_{k}=x_{k}, \quad k=1, \cdots, m,
$$


where $\left\{e_{1}, \cdots, e_{n}\right\}$ is the standard orthonormal basis of $\mathbf{C}^{n}$, and compute that

$$
\begin{aligned}
\left(C_{m}(A) x^{\wedge}, x^{\wedge}\right) & =\left(C_{m}(A) C_{m}(U) e_{1} \wedge \cdots \wedge e_{m}, C_{m}(U) e_{1} \wedge \cdots \wedge e_{m}\right) \\
& =\left(C_{m}\left(U^{*} A U\right) e_{1} \wedge \cdots \wedge e_{m}, e_{1} \wedge \cdots \wedge e_{m}\right) \\
& =\operatorname{det}\left(U^{*} A U\right)[1, \cdots, m \mid 1, \cdots, m]
\end{aligned}
$$

where $\left(U^{*} A U\right)[1, \cdots, m \mid 1, \cdots, m]$ indicates the submatrix of $U^{*} A U$ lying in rows and columns $1, \cdots, m$. In view of (6), this yields yet another formulation of the $m$ th decomposable numerical range: denoting by $U_{n}(C)$ the multiplicative group of $n$-square unitary matrices, we have

$$
W_{m}^{\wedge}(A)=\left\{\operatorname{det}\left(U^{*} A U\right)[1, \cdots, m \mid 1, \cdots, m] \mid U \in U_{n}(\mathbf{C})\right\} .
$$

From (6) we obtain

$$
W_{m}^{\wedge}(A) \subset W\left(C_{m}(A)\right)
$$

and hence

$$
r_{m}^{\wedge}(A) \leqq r\left(C_{m}(A)\right)
$$

Strict inequality may hold in (9); e.g., consider

$$
A=\left[\begin{array}{llll}
0 & 0 & 1 & 0 \\
0 & 0 & 0 & 1 \\
0 & 0 & 0 & 0 \\
0 & 0 & 0 & 0
\end{array}\right] \in M_{4}(\mathbf{C})
$$

with $m=2[1]$.

We define $P_{m}(A)$, the $m$ th decomposable eigenpolygon of $A$, to be the convex polygon in the complex plane spanned by all products of $m$ eigenvalues of $A$. Thus

$$
P_{m}^{\wedge}(A)=\mathscr{H}\left(\left\{\prod_{k=1}^{m} \lambda_{\omega(k)} \mid \omega \in Q_{m, n}\right\}\right)
$$

where $\lambda_{1}, \cdots, \lambda_{n}$ are the eigenvalues of $A, \mathscr{H}$ denotes convex hull, and $Q_{m, n}$ is the set of all strictly increasing sequences of $m$ integers chosen from $\{1, \cdots, n\}$. When $m=1, P_{1}(A)$ is simply written $P(A)$ and called the eigenpolygon of $A$. It should be observed that the sets $W_{m}^{\wedge}(A)$ and $P_{m}^{\wedge}(A)$ are both invariant under transformation of $A$ by a unitary similarity, that is, 


$$
W_{m}^{\wedge}\left(U^{*} A U\right)=W_{m}^{\wedge}(A)
$$

and

$$
P_{m}^{\wedge}\left(U^{*} A U\right)=P_{m}^{\wedge}(A)
$$

for any $U \in U_{n}(\mathbf{C})$.

Proposition 1. Let $A \in M_{n}(\mathbf{C})$ have eigenvalues $\lambda_{1}, \cdots, \lambda_{n}$, and let $m \in\{1, \cdots, n\}$. Then

$$
\prod_{k=1}^{m} \lambda_{\omega(k)} \in W_{m}^{\wedge}(A), \omega \in Q_{m, n}
$$

Moreover, if $A$ is normal then

$$
W_{m}^{\wedge}(A) \subset P_{m}^{\wedge}(A)
$$

Proof. Fix $\omega \in Q_{m, n}$. By the Schur triangularization theorem, there exists a matrix $U \in U_{n}(\mathbf{C})$ such that $U^{*} A U$ is an upper triangular matrix with first $m$ main diagonal elements $\lambda_{\omega(1)}, \cdots, \lambda_{\omega(m)}$. Then

$$
\prod_{k=1}^{m} \lambda_{\omega(k)}=\operatorname{det}\left(U^{*} A U\right)[1, \cdots, m \mid 1, \cdots, m]
$$

In view of $(7),(11)$ is established.

Next, assume $A \in M_{n}(C)$ is normal. Let $\left\{u_{1}, \cdots, u_{n}\right\}$ be an orthonormal basis of $\mathbf{C}^{n}$ such that

$$
A u_{i}=\lambda_{i} u_{i}, \quad i=1, \cdots, n
$$

Then

$$
\left\{u_{\omega}^{\wedge}=u_{\omega(1)} \wedge \cdots \wedge u_{\omega(m)} \in G_{m}\left(\mathbf{C}^{n}\right) \mid \omega \in Q_{m, n}\right\}
$$

is an orthonormal basis of $\wedge^{m} C^{n}\left[3\right.$, p. 132]. Given $x^{\wedge} \in G_{m}\left(C^{n}\right)$, we have

$$
\left(C_{m}(A) x^{\wedge}, x^{\wedge}\right)=\left(C_{m}(A) \sum_{\omega \in Q_{m, n}}\left(x^{\wedge}, u_{\omega}^{\wedge}\right) u_{\omega}^{\wedge}, \sum_{\omega \in Q_{m, n}}\left(x^{\wedge}, u_{\omega}^{\wedge}\right) u_{\omega}^{\wedge}\right)
$$

$$
=\sum_{\omega \in Q_{m, n}}\left|\left(x^{\wedge}, u_{\omega}^{\wedge}\right)\right|^{2} \prod_{k=1}^{m} \lambda_{\omega(k)} .
$$

Since

$$
\sum_{\omega \in Q_{m, n}}\left|\left(x^{\wedge}, u_{\omega}^{\wedge}\right)\right|^{2}=\left\|x^{\wedge}\right\|^{2}=1
$$


(13) expresses the element $\left(C_{m}(A) x^{\wedge}, x^{\wedge}\right)$ of $W_{m}^{\wedge}(A)$ as a convex combination of all products of $m$ eigenvalues of $A$. This establishes (12).

CoRollary 1. Let $A \in M_{n}(\mathbf{C})$ be normal and $m \in$ $\{1, \cdots, n\}$. Then $r_{m}^{\wedge}(A)$ is the maximum modulus of a product of $m$ eigenvalues of $A$.

Corollary 2. Let $A=c Z \in M_{n}(\mathbf{C})$, where $Z \in U_{n}(\mathbf{C})$ and $c \in \mathbf{C}$, and let $m \in\{1, \cdots, n\}$. Then

$$
r_{m}^{\wedge}(U A V)=r_{m}^{\wedge}(A)
$$

for all $U, V \in U_{n}(\mathbf{C})$.

3. Some lemmas. In the following discussion let $A \in M_{n}(C)$ be a fixed matrix, $m \in\{1, \cdots, n\}$ a fixed positive integer, and assume the rank of $A$ is at least $m$. Denote the singular values of $A$ by $\alpha_{1}, \cdots, \alpha_{n}$, arranged so that

$$
\alpha_{1} \geqq \cdots \geqq \alpha_{n} \geqq 0,
$$

and set

$$
D=\operatorname{diag}\left(\alpha_{1}, \cdots, \alpha_{n}\right) \in M_{n}(\mathbf{C}) .
$$

It is well known that there exist matrices $U_{1}, V_{1} \in U_{n}(\mathbf{C})$ such that

$$
A=U_{1} D V_{1}
$$

Suppose momentarily that

$$
r_{m}^{\wedge}(U A V)=r_{m}^{\wedge}(A)
$$

for all $U, V \in U_{n}(\mathrm{C})$. Then clearly

$$
r_{m}^{\wedge}(U D V)=r_{m}^{\wedge}(D)
$$

for all $U, V \in U_{n}(\mathbf{C})$ :

$$
\begin{aligned}
r_{m}^{\wedge}(U D V) & =r_{m}^{\wedge}\left(U U_{1}^{*} A V_{1}^{*} V\right) \\
& =r_{m}^{\wedge}(A) \\
& =r_{m}^{\wedge}\left(U_{1}^{*} A V_{1}^{*}\right) \\
& =r_{m}^{\wedge}(D) .
\end{aligned}
$$


Fix $U_{0} \in U_{n}(\mathrm{C})$ and choose $x_{0} \in G_{m}\left(C^{n}\right)$ so that

$$
r_{m}^{\wedge}\left(U_{0} D\right)=\left|\left(C_{m}\left(U_{0} D\right) x_{0}^{\wedge}, x_{\hat{0}}\right)\right| .
$$

Set

$$
y_{0}^{\hat{0}}=C_{m}\left(U_{0}^{*}\right) x_{\hat{0}} \in G_{m}\left(\mathbf{C}^{n}\right)
$$

Let $\left\{e_{1}, \cdots, e_{n}\right\}$ be the standard orthonormal basis of $\mathbf{C}^{n}$; then

$$
\left\{e_{\omega}^{\wedge}=e_{\omega(1)} \wedge \cdots \wedge e_{\omega(m)} \in G_{m}\left(C^{n}\right) \mid \omega \in Q_{m, n}\right\}
$$

is the induced orthonormal basis of $\wedge^{m} C^{n}$. Write

$$
x_{\hat{0}}=\sum_{\omega \in Q_{m, n}} \chi_{\omega} e_{\omega,}^{\wedge} \chi_{\omega} \in \mathrm{C}, \omega \in Q_{m, n}
$$

and

$$
y_{0}^{\wedge}=\sum_{\omega \in Q_{m, n}} \eta_{\omega} e_{\omega}^{\wedge}, \eta_{\omega} \in \mathbf{C}, \omega \in Q_{m, n}
$$

Lemma 1. Assume

$$
r_{m}^{\wedge}\left(U_{0} D\right)=r_{m}^{\wedge}(D)
$$

Then

$$
\alpha_{1} \cdots \alpha_{m}=\alpha_{\omega(1)} \cdots \alpha_{\omega(m)}
$$

for every $\omega \in Q_{m, n}$ for which $\chi_{\omega} \neq 0$. Moreover,

$$
\left|\chi_{\omega}\right|=\left|\eta_{\omega}\right|, \omega \in Q_{m, n}
$$

Proof. Notice that

$$
\alpha_{1} \cdots \alpha_{m}>0
$$

since $A$ has rank at least $m$. We compute

$$
\begin{array}{rlrl}
\alpha_{1} \cdots \alpha_{m} & =r_{m}^{\wedge}(D) & & \text { (by Corollary 1) } \\
& =r_{m}^{\wedge}\left(U_{0} D\right) & & \text { (by hypothesis) } \\
& =\left|\left(C_{m}\left(U_{0} D\right) x_{0}^{\wedge}, x_{0}^{\wedge}\right)\right|(\text { by (16)) }
\end{array}
$$




$$
=\left|\sum_{\omega \in Q_{m, n}} \alpha_{\omega} \chi_{\omega} \bar{\eta}_{\omega}\right| \quad\left(\alpha_{\omega}=\alpha_{\omega(1)} \cdots \alpha_{\omega(m)}\right)
$$

(20)

$$
\begin{aligned}
& \leqq \sum_{\omega \in Q_{m, n}} \alpha_{\omega}\left|\chi_{\omega} \| \eta_{\omega}\right| \\
& \leqq \alpha_{1} \cdots \alpha_{m} \sum_{\omega \in Q_{m, n}}\left|\chi_{\omega} \| \eta_{\omega}\right| \\
& \leqq \alpha_{1} \cdots \alpha_{m}\left(\sum_{\omega \in Q_{m, n}}\left|\chi_{\omega}\right|^{2}\right)^{\frac{1}{2}}\left(\sum_{\omega \in O_{m, n}}\left|\eta_{\omega}\right|^{2}\right)^{\frac{1}{2}} \\
& =\alpha_{1} \cdots \alpha_{m}\left\|x_{\hat{0}}\right\|\left\|y_{\hat{0}}\right\| \\
& =\alpha_{1} \cdots \alpha_{m} .
\end{aligned}
$$

The last inequality in (20) is the Cauchy-Schwarz inequality. Since equality holds throughout, $\alpha_{1} \cdots \alpha_{m}>0$, and $x_{\hat{0}}, y_{\hat{0}} \neq 0$, we conclude that

$$
\left|\chi_{\omega}\right|=c\left|\eta_{\omega}\right|, \omega \in Q_{m, n}
$$

for some $c>0$. But then $\left\|x_{\hat{0}}\right\|=1=\left\|y_{\hat{0}}\right\|$ implies $c=1$. Thus

$$
\left|\chi_{\omega}\right|=\left|\eta_{\omega}\right|, \omega \in Q_{m, n}
$$

It follows from equality in the second inequality in (20) that

$$
\alpha_{1} \cdots \alpha_{m}=\alpha_{\omega(1)} \cdots \alpha_{\omega(m)}
$$

for -every $\omega \in Q_{m, n}$ for which $\chi_{\omega} \neq 0$.

Suppose now that $\sigma$ is a permutation in $S_{n}$, the symmetric group of degree $n$, and $U_{0}^{*} \in U_{n}(\mathbf{C})$ is the permutation matrix corresponding to $\sigma$ :

$$
U_{0}^{*}=P(\sigma)=\left[\delta_{i \sigma())}\right] \text {. }
$$

In this situation, continuing with the above notation, we have

$$
\begin{aligned}
y_{0}^{\hat{0}} & =C_{m}(P(\sigma)) x_{\hat{0}} \\
& =\sum_{\omega \in Q_{m, n}} X_{\omega} C_{m}(P(\sigma)) e_{\omega}^{\hat{\omega}} \\
& =\sum_{\omega \in Q_{m, n}} \chi_{\omega} e_{\sigma \omega(1)} \wedge \cdots \wedge e_{\sigma \omega(m)}\left(\text { since } P(\sigma) e_{i}=e_{\sigma(i)}, i=1, \cdots, n\right) \\
& =\sum_{\omega \in Q_{m, n}} \epsilon_{\omega} \chi_{\omega} e_{\omega_{\omega}} .
\end{aligned}
$$


Here $\omega_{\sigma} \in Q_{m, n}$ is the strictly increasing rearrangement of the sequence

$$
(\sigma \omega(1), \cdots, \sigma \omega(m))
$$

and $\epsilon_{\omega}= \pm 1$ is the sign of the permutation

$$
\left(\begin{array}{c}
\sigma \omega(1) \cdots \sigma \omega(m) \\
\omega_{\sigma}(1) \cdots \omega_{\sigma}(m)
\end{array}\right)
$$

The mapping

$$
\omega \mapsto \omega_{\sigma}, \omega \in Q_{m, n}
$$

is clearly a bijection of $Q_{m, n}$. Hence from (19) and (21),

$$
\begin{aligned}
y_{\hat{0}} & =\sum_{\omega \in Q_{m, n}} \eta_{\omega} e_{\omega}^{\hat{\omega}} \\
& =\sum_{\omega \in Q_{m, n}} \eta_{\omega \sigma} e_{\omega_{\sigma}} \\
& =\sum_{\omega \in Q_{m, n}} \epsilon_{\omega} \chi_{\omega} e_{\omega_{\sigma}}^{\hat{n}}
\end{aligned}
$$

so that

$$
\eta_{\omega \sigma}=\epsilon_{\omega} X_{\omega}, \omega \in Q_{m, n}
$$

Lemma 2. Assume

$$
r_{m}^{\wedge}\left(P(\sigma)^{T} D\right)=r_{m}^{\wedge}(D)
$$

Then

$$
\alpha_{1} \cdots \alpha_{m}=\alpha_{\omega_{\sigma}(1)} \cdots \alpha_{\omega_{\sigma}(m)}
$$

for every $\omega \in Q_{m, n}$ for which $\chi_{\omega_{0}} \neq 0$. Moreover,

$$
\left|\chi_{\omega_{0}}\right|=\left|\chi_{\omega}\right|, \omega \in Q_{m, n}
$$

Proof. The first assertion is immediate from Lemma 1, as is the second:

$$
\begin{aligned}
\left|\chi_{\omega_{o}}\right| & =\left|\eta_{\omega_{o}}\right| \\
& =\left|\epsilon_{\omega} \chi_{\omega}\right| \quad(\text { by (22)) } \\
& =\left|\chi_{\omega}\right|, \omega \in Q_{m, n \cdot}
\end{aligned}
$$




\section{The main result.}

TheOREM 1. Let $A \in M_{n}(C)$ and let $m$ be a positive integer, $1 \leqq$ $m<n$. Assume the rank of $A$ is at least $m$. Then

$$
r_{m}^{\wedge}(U A V)=r_{m}^{\wedge}(A)
$$

for all $U, V \in U_{n}(\mathbf{C})$ if and only if $A$ is a scalar multiple of a unitary matrix.

Proof. We have observed in Corollary 2 that the condition is sufficient.

To see that the condition is necessary, assume (23) holds for all $U, V \in U_{n}(\mathbf{C})$. Since there exist matrices $U_{1}, V_{1} \in U_{n}(\mathbf{C})$ such that

$$
A=U_{1} D V_{1}
$$

where

$$
D=\operatorname{diag}\left(\alpha_{1}, \cdots, \alpha_{n}\right) \in M_{n}(\mathbf{C})
$$

and

$$
\alpha_{1} \geqq \cdots \geqq \alpha_{n}
$$

are the singular values of $A$, it suffices to show that

$$
\alpha_{1}=\alpha_{n}
$$

Consider the full cycle

$$
\varphi=(12 \cdots n) \in S_{n}
$$

Choose $x_{0}^{\wedge} \in G_{m}\left(C^{n}\right)$ so that

$$
r_{m}^{\wedge}\left(P(\varphi)^{T} D\right)=\left|\left(C_{m}\left(P(\varphi)^{T} D\right) x_{\hat{0}}, x_{0}^{\hat{0}}\right)\right|
$$

and write

$$
x_{\hat{0}}^{\hat{n}}=\sum_{\omega \in Q_{m, n}} \chi_{\omega} e_{\hat{\omega},}^{\hat{n}} \chi_{\omega} \in \mathbf{C}, \omega \in Q_{m, n}
$$

Since

$$
\sum_{\omega \in Q_{m, n}}\left|\chi_{\omega}\right|^{2}=\left\|x_{0}\right\|^{2}=1
$$


there exists $\omega \in Q_{m, n}$ for which

$$
\chi_{\omega} \neq 0 \text {. }
$$

Set

$$
\gamma=\omega_{{ }_{\varphi} n-\omega(1)+1} \in Q_{m, n}
$$

By (15) and Lemma 2 (with $\sigma=\varphi^{n-\omega(1)+1}$ ), $\left|\chi_{\gamma}\right|=\left|\chi_{\omega}\right|$ and hence by (24)

$$
\chi_{y} \neq 0 \text {. }
$$

Also observe that

$$
\begin{aligned}
\varphi^{n-\omega(1)+1} \omega(1) & =\varphi(\omega(1)+n-\omega(1)) \\
& =\varphi(n) \\
& =1
\end{aligned}
$$

implies $\omega_{\varphi n-\omega(1)+1}(1)=1$, i.e.,

$$
\gamma(1)=1
$$

The argument now splits into two cases.

Case I. $\gamma(m)<n$. Apply the permutation $\varphi^{n-\gamma(m)}$ to

$$
\gamma=(1, \gamma(2), \cdots, \gamma(m))
$$

to obtain

$$
\varphi^{n-\gamma(m)} \gamma=(1+n-\gamma(m), \gamma(2)+n-\gamma(m), \cdots, \gamma(m-1)+n-\gamma(m), n)
$$

$$
=\gamma_{\varphi n-\gamma(m)}
$$

Since $\gamma(m)<n$, we have

$$
\begin{aligned}
& 2 \leqq 1+n-\gamma(m) \\
& 3 \leqq \gamma(2)+n-\gamma(m) \\
& \quad \vdots \\
& m \leqq \gamma(m-1)+n-\gamma(m)
\end{aligned}
$$


Therefore

$$
\alpha_{2} \alpha_{3} \cdots \alpha_{m} \geqq \alpha_{1+n-\gamma(m)} \alpha_{\gamma(2)+n-\gamma(m)} \cdots \alpha_{\gamma(m-1)+n-\gamma(m)} .
$$

By (15) and Lemma 2 (with $\left.\sigma=\varphi^{n-\gamma(m)}\right),\left|\chi_{\gamma_{\varphi n-\gamma(m)}}\right|=\left|\chi_{\gamma}\right|$ and hence by (26)

$$
\chi_{\gamma_{\varphi n-\gamma(m)}} \neq 0
$$

Then Lemma 2 together with (27) implies

$$
\alpha_{1} \alpha_{2} \alpha_{3} \cdots \alpha_{m}=\alpha_{1+n-\gamma(m)} \alpha_{\gamma(2)+n-\gamma(m)} \cdots \alpha_{\gamma(m-1)+n-\gamma(m)} \alpha_{n}
$$

Since $\alpha_{1} \cdots \alpha_{m}>0$ ( $A$ has rank at least $m$ ), it follows from (28) and (29) that

$$
\alpha_{1}=\alpha_{n}
$$

Case II. $\gamma(m)=n$. In this case

$$
\gamma=(1, \gamma(2), \cdots, \gamma(m-1), n) .
$$

Now $m<n$ by hypothesis, so there exists a least positive integer $k \in\{2, \cdots, m\}$ such that

$$
k<\gamma(k) \text {. }
$$

Apply the permutation $\varphi^{1-k}$ to

$$
\gamma=(1, \cdots, k-1, \gamma(k), \cdots, \gamma(m-1), n)
$$

to obtain

$$
\begin{array}{r}
\varphi^{1-k} \gamma=(n-k+2, n-k+3, \cdots, n-1, n, \gamma(k)-k+1, \cdots, \\
\gamma(m-1)-k+1, n-k+1)
\end{array}
$$

Then

$$
\begin{array}{r}
\gamma_{\varphi^{1-k}}=(\gamma(k)-k+1, \cdots, \gamma(m-1)-k+1, n-k+1, n-k+2, \\
n-k+3, \cdots, n-1, n) .
\end{array}
$$

Since $k<\gamma(k)$, we have 


$$
\begin{aligned}
2 & \leqq \gamma(k)-k+1, \\
3 & \leqq \gamma(k+1)-k+1, \\
& \vdots \\
m-k+1 & \leqq \gamma(m-1)-k+1, \\
m-k+2 & \leqq n-k+1, \\
m-k+3 & \leqq n-k+2, \\
& \vdots \\
m & \leqq n-1 .
\end{aligned}
$$

Therefore

$$
\alpha_{2} \alpha_{3} \cdots \alpha_{m} \geqq \alpha_{\gamma(k)-k+1} \alpha_{\gamma(k+1)-k+1} \cdots \alpha_{n-1} .
$$

By (15) and Lemma 2 (with $\sigma=\varphi^{1-k}$ ), $\left|\chi_{\gamma_{\varphi 1-k}}\right|=\left|\chi_{\gamma}\right|$ and hence by (26)

$$
\chi_{\gamma_{\varphi 1-k}} \neq 0
$$

Then Lemma 2 together with (30) implies

$$
\alpha_{1} \alpha_{2} \alpha_{3} \cdots \alpha_{m}=\alpha_{\gamma(k)-k+1} \alpha_{\gamma(k+1)-k+1} \cdots \alpha_{n-1} \alpha_{n}
$$

Once again, since $\alpha_{1} \cdots \alpha_{m}>0$ it follows from (31) and (32) that

$$
\alpha_{1}=\alpha_{n}
$$

This completes the proof.

We remark that the restriction $m \neq n$ in Theorem 1 is inevitable. Indeed, for any matrix $A \in M_{n}(C)$,

$$
\begin{aligned}
\boldsymbol{r}_{n}^{\wedge}(A) & =|\operatorname{det}(A)| \\
& =|\operatorname{det}(U A V)| \\
& =r_{n}^{\wedge}(U A V)
\end{aligned}
$$

for all $U, V \in U_{n}(\mathrm{C})$. The hypothesis that $A$ have rank at least $m$ is equally essential, since any matrix $A \in M_{n}(C)$ of rank less than $m$ satisfies

$$
r_{m}^{\wedge}(A)=0=r_{m}^{\wedge}(U A V)
$$

for all $U, V \in U_{n}(\mathbf{C})$. 


\section{REFERENCES}

1. P. Andresen and M. Marcus, Weyl's inequality and quadratic forms on the Grassmannian, Pacific J. Math., 67 (1976), 277-289.

2. P. R. Halmos, A Hilbert Space Problem Book, Van Nostrand Co., Inc. (1967).

3. M. Marcus, Finite Dimensional Multilinear Algebra, Part I, Marcel Dekker, Inc. (1973).

4. —, Finite Dimensional Multilinear Algebra, Part II, Marcel Dekker, Inc. (1975).

Received May 5, 1977. The research of the second author was supported by the Air Force Office of Scientific Research under Grant AFOSR 77-3166.

INSTITUTE FOR ALGEBRA AND COMBINATORICS

UNIVERSITY OF CALIFORNIA

SANTA BARBARA, CA 93106 



\section{PACIFIC JOURNAL OF MATHEMATICS}

\section{EDITORS}

RICHARD ARENS (Managing Editor)

University of California

Los Angeles, CA 90024

R. A. BeAumont

University of Washington

Seattle, WA 98105

C. C. MOORE

University of California

Berkeley, CA 94720
J. DUGUNDJI

Department of Mathematics

University of Southern California

Los Angeles, CA 90007

R. FINN AND J. MILGRAM

Stanford University

Stanford, CA 94305

\section{ASSOCIATE EDITORS}
E. F. BECKENBACH
B. H. NEUMANN
F. WOLF
K. YoshidA

\section{SUPPORTING INSTITUTIONS}

UNIVERSITY OF BRITISH COLUMBIA

UNIVERSITY OF SOUTHERN CALIFORNIA

CALIFORNIA INSTITUTE OF TECHNOLOGY

STANFORD UNIVERSITY

UNIVERSITY OF CALIFORNIA

UNIVERSITY OF HAWAII

MONTANA STATE UNIVERSITY

UNIVERSITY OF TOKYO

UNIVERSITY OF NEVADA

UNIVERSITY OF UTAH

NEW MEXICO STATE UNIVERSITY

OREGON STATE UNIVERSITY

UNIVERSITY OF OREGON

OSAKA UNIVERSITY

WASHINGTON STATE UNIVERSITY

UNIVERSITY OF WASHINGTON

AMERICAN MATHEMATICAL SOCIETY

The Supporting Institutions listed above contribute to the cost of publication of this Journal, but they are not owners or publishers and have no responsibility for its contents or policies.

Mathematical papers intended for publication in the Pacific Journal of Mathematics should be in typed form or offset-reproduced (not dittoed), double spaced with large margins. Underline Greek letters in red, German in green, and script in blue. The first paragraph or two must be capable of being used separately as a synopsis of the entire paper. Items of the bibliography should not be cited there unless absolutely necessary, in which case they must be identified by author and Journal, rather than by item number. Manuscripts, in duplicate, may be sent to any one of the four editors. Please classify according to the scheme of Math. Reviews, Index to Vol. 39. All other communications should be addressed to the managing editor, or Elaine Barth, University of California, Los Angeles, California, 90024.

100 reprints are provided free for each article, only if page charges have been substantially paid. Additional copies may be obtained at cost in multiples of 50 .

The Pacific Journal of Mathematics is issued monthly as of January 1966. Regular subscription rate: $\$ 72.00$ a year (6 Vols., 12 issues). Special rate: $\$ 36.00$ a year to individual members of supporting institutions.

Subscriptions, orders for numbers issued in the last three calendar years, and changes of address should be sent to Pacific Journal of Mathematics, 103 Highland Boulevard, Berkeley, California, 94708.

PUBLISHED BY PACIFIC JOURNAL OF MATHEMATICS, A NON-PROFIT CORPORATION

Printed at Jerusalem Academic Press, POB 2390, Jerusalem, Israel. 


\section{Pacific Journal of Mathematics}

\section{Vol. 75, No. $2 \quad$ October, 1978}

Susan Jane Zimmerman Andima and W. J. Thron, Order-induced

topological properties ................................... 297

Gregory Wade Bell, Cohomology of degree 1 and 2 of the Suzuki groups . . 319

Richard Body and Roy Rene Douglas, Rational homotopy and unique

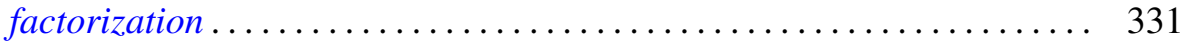

Frank Lewis Capobianco, Fixed sets of involutions ................. 339

L. Carlitz, Some theorems on generalized Dedekind-Rademacher sums .... 347

Mary Rodriguez Embry and Alan Leslie Lambert, The structure of a special class of weighted translation semigroups .....................

Steve Ferry, Strongly regular mappings with compact ANR fibers are

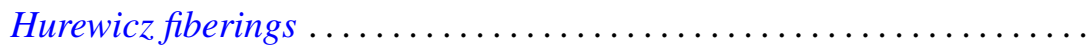

Ivan Filippenko and Marvin David Marcus, On the unitary invariance of the

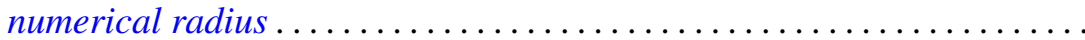

$\mathrm{H}$. Groemer, On the extension of additive functionals on classes of convex

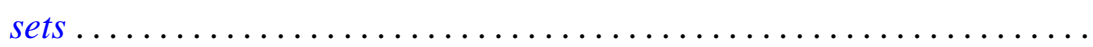

Rita Hall, On the cohomology of Kuga's fiber variety ............... 411

H. B. Hamilton, Congruences on $\mathrm{N}$-semigroups ................. 423

Manfred Herrmann and Rolf Schmidt, Regular sequences and lifting

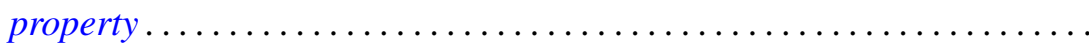

James Edgar Keesling, Decompositions of the Stone-Čech compactification which are shape equivalences .....................

Michael Jay Klass and Lawrence Edward Myers, On stopping rules and the expected supremum of $S_{n} / T_{n}$

Ronald Charles Linton, $\lambda$-large subgroups of $C_{\lambda}$-groups

William Owen Murray, IV and L. Bruce Treybig, Triangulations with the free cell property ............................

Louis Jackson Ratliff, Jr., Polynomial rings and $H_{i}$-local rings ...

Michael Rich, On alternate rings and their attached Jordan rings....

Gary Sampson and H. Tuy, Fourier transforms and their Lipschitz

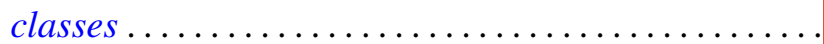

Helga Schirmer, Effluent and noneffluent fixed points on dendrites ...

Daniel Byron Shapiro, Intersections of the space of skew-symmetric maps

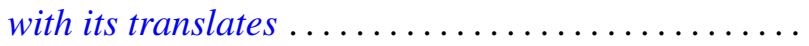

Edwin Spanier, Tautness for Alexander-Spanier cohomology ...

Alan Stein and Ivan Ernest Stux, A mean value theorem for binary digits ...

Franklin D. Tall, Normal subspaces of the density topology . .

William Yslas Vélez, Prime ideal decomposition in $F\left(\mu^{1 / p}\right) \ldots$ 